\title{
INVESTIGACIÓN/RESEARCH
}

\section{LA COMUNICACIÓN PARA LA SALUD Y SUS APORTES DESDE EL MODELO DE PENDER: COMUNICACIÓN Y CULTURA RELIGIOSA}

John Esteban Carreño-Salgado ${ }^{1}$ : Universidad Autónoma de Barcelona. España johncarreo@yahoo.es

Carlos Felimer Del Valle-Rojas: Universidad de La Frontera. Chile delvalle@ufro.cl

Ramón Cladellas-Pross: Universidad Autónoma de Barcelona. España ramon.cladellas@uab.es

Daniza María Ivanovic-Marincovic: Universidad de Chile. Chile daniza@inta.cl

\section{RESUMEN}

El objetivo de este estudio fue determinar el efecto a largo plazo de un programa de promoción de salud (PPS) sobre el comportamiento de mujeres Adventistas del Séptimo Día (MASD) y mujeres No Adventistas del Séptimo Día (MNASD). La intervención contempló un Programa Promotor de Estilo de Vida saludable (PPEVS). EI programa está basado en el Modelo de Pender y mide seis dimensiones: Autorrealización, Responsabilidad en Salud, Ejercicio Físico, Nutrición, Apoyo Interpersonal y Manejo de Estrés. El propósito de este estudio fue determinar la efectividad de la estrategia comunicativa, con la cual se implantó e implementó en la comunidad, la intervención educativa relativa a evaluar el PPEVS. El estudio inicial, de naturaleza experimental y de cohorte única y comparativo, se llevo a cabo el año 2002 en la ciudad de Villarrica. El análisis del impacto del PPEVS después de seis años mostró un nivel alto de eficacia de la intervención comunicativa usada en el programa en las MASD. Concluimos que la estrategia comunicativa usada en el programa fue importante para los cambios positivos de conducta durante 2002 .Se observó un

\footnotetext{
${ }^{1}$ Autor Correspondiente

John Esteban Carreño-Salgado: Departamento de Psicología Básica, evolutiva y de Educación, Universidad Autónoma de Barcelona. España

Correo: johncarreo@yahoo.es
} 
descenso significativo en las medianas del EVPS de 3,1 a 2,7 ( $P<0.001$ ). Este estudio determino la importancia de refuerzo permanente de comportamientos. En todo el período de tiempo post intervención, las MASD dieron continuidad al estilo de vida adoptado a través de la práctica de la religión, las MASD mantuvieron las conductas promotoras de vida saludable.

PALABRAS CLAVE: Promoción de salud - Estilo de vida - Comportamiento de salud Estrategia de comunicación

\title{
THE COMMUNICATION FOR THE HEALTH AND HIS CONTRIBUTIONS FROM THE PENDER MODEL: COMMUNICATION AND RELIGIOUS CULTURE
}

\begin{abstract}
The aim of this study was to determine the long-term effect of a health promotion program (HPP) on behaviour in Seventh- Day Adventist church Women (SDAW) and Non-Seventh-Day Adventist Church Women (NSDAW) as measured by the Health-Promoting Lifestyle Profile (HPLP). The HPLP is based on Pender's Model measures six dimensions: Self- actualization, Responsibility in Health, Exercise, Nutrition, Interpersonal Support and Stress Management. The initial study, conducted in 2002, was an experimental, comparative single cohort design in the town of Villarrica County, Ninth Region of Chile. The study reported here compared the results obtained in HPL of the 2002 post-test and the re-test in 2008, among Seventh-Day Adventist Church women (SDAW), and non-Seventh-Day Adventist Church women (NSDAW). Follow-up and comparative study between HPP results in the post-test period in 2002 and re-test periods in 2008, six years after HPP was finished. In both study periods, an instrument was applied to measure the HPLP in both groups. Analyzing the impact of HPLP after six years showed a high level of efficiency of the communicative strategy of the program in the SDAW. We conclude that the communicative strategy used in the intervention was important for the positive changes during 2002 in which people adopted behaviours that promote healthful living, both in SDAW and in some dimensions of HPLP among NSDAW. The NSDAW showed a significant decrease in some dimensions of the HPLP.
\end{abstract}

KEY WORDS: Health promotion - Health education - Lifestyle, Health behaviour Communication strategy 


\section{1.- INTRODUCCIÓN}

El lograr la salud de las poblaciones ha sido preocupación prioritaria en todas las épocas. Para alcanzar este bienestar de tanta relevancia para el desarrollo humano, se han buscado diferentes enfoques, para organizar la atención de salud y lograr el impacto necesario. Inicialmente, se centraban los modelos de atención principalmente en las estrategias para recuperar la salud perdida, y luego se dio mayor relevancia a las estrategias orientadas hacia la prevención de las enfermedades y la promoción de la salud. La necesidad de variar los enfoques para conseguir y mantener la salud se debe a los cambios demográficos, epidemiológicos, socioeconómicos, de las comunicaciones y las condiciones de vida que han experimentado las poblaciones, para reconocer los factores de riesgos emergentes que han hecho variar las necesidades en salud. (OPS, 1996)

El interés por entender lo que motiva a las personas a adoptar o no adoptar comportamientos que mejorarán su calidad de vida ha sido un tema de investigación para muchas disciplinas. Entre las teorías usadas con frecuencia para explicar este proceso se encuentran las relacionadas con los modelos de las etapas del cambio de comportamiento y las teorías de la persuasión, que pueden aplicarse a distintas culturas y a distintos comportamientos relacionados con la salud. La adopción de comportamientos sanos es un proceso en el cual los individuos avanzan, a través de diversas etapas, hasta que el nuevo comportamiento se convierta en parte de la vida diaria. El público destinatario se segmenta para identificar grupos más amplios de personas que comparten valores similares, tienen las mismas creencias o tienen en común otros atributos claves que repercutirán en su atención y en su respuesta a la información sanitaria. Los programas de comunicación para la salud se planifican, se ejecutan y se evalúan después de un proceso sistemático. Un ejemplo de esto es que la División de Promoción y Protección de la Salud de la OPS lo utiliza, con la intención de comprender las percepciones, las creencias, los valores y las prácticas de la población en riesgo, para desarrollar programas de comunicación que atraigan y persuadan al público destinatario a que adopte modos de vida sanos.

La promoción de la salud busca desarrollar tanto el potencial del individuo como de su familia, la comunidad, la sociedad y también del medioambiente, entendiendo éste como las circunstancias físicas, interpersonales y económicas en que se desempeña la persona. Es una aproximación proactiva a la salud que incluye el desarrollo de fuerzas, incremento de recursos y fomento de la resiliencia con el fin de lograr el funcionamiento óptimo del ser humano. ( Pender, Murdaugh, Parson, 2001; Jauregui, Suarez, 1998).

Los países han realizado esfuerzos sostenidos para promover dietas y estilos de vida saludables basados en las recomendaciones de la OMS. En este contexto, los hallazgos de varios autores sugieren la necesidad de implementar más programas educativos enfocados a prevenir enfermedades cardiovasculares, obesidad, diabetes y cáncer, especialmente para aquellos que viven en la pobreza. (FAO/OMS. 1992; Arthur, Pena, Holbert, 2001; Burdine, Chen, Gottlieb, Peterson, Demetri Vacalis, 1984; Reynolds, Raczynski, Binkley, Franklin, Duvall, Devane-Hart, 1998 ; Reynolds, 
Franklin, Binkley, Raczynski, Harrington, Kirk, 2000; Donnelly, Jacobsen, Whatley, Hill, Swift, Cherrington, 1996)

Para mejorar la calidad de vida de la población, la OMS ha planteado que la promoción de salud es la estrategia fundamental de la atención primaria. Esta estrategia implica la modificación de conductas en las personas: abandonar las que perjudican la salud y adoptar las que la favorecen. Sin embargo, antecedentes teóricos confirman que, para que el cambio de conducta se logre, se requiere de un proceso cognitivo-perceptual en el que destaca que la persona tenga un motivo real para el cambio; de lo contrario, el plan de promoción no obtendrá buenos resultados.

De esta manera y considerando que la mujer cumple un rol fundamental en las generaciones futuras, específicamente en el desarrollo de valores, actitudes y conductas capaces de reducir situaciones de riesgo que constituirán el estilo de vida de la familia y de la comunidad, es que se realizó el año 2002, una intervención educativa que consistió en un Programa de Estilo de Vida Promotor de Salud. Este programa estuvo dirigido al auto cuidado en salud, usando el modelo de promoción de salud de Pender, (Pender, et al., 2001) por sus investigaciones desarrolladas en esta área, basados en la Teoría Cognitiva Social de Bandura, (Jauregui, et al., 1998) y la teoría de la expectativa de valor que le han permitido desarrollar un modelo que permite explicar, predecir y modificar conductas promotoras de salud.

Las conductas promotoras de salud integradas al estilo de vida saludable permiten mejorar la salud, perfeccionar el funcionamiento y generar una mejor calidad de vida en cualquier etapa del desarrollo. (Pender, et al., 2001) Las teorías o los modelos del cambio de comportamiento postulan que la adopción de comportamientos sanos es un proceso en el cual los individuos avanzan, a través de diversas etapas, hasta que el nuevo comportamiento se convierta en parte de la vida diaria.

\subsection{Definición y justificación del problema}

En las últimas décadas, la transición demográfica y epidemiológica ha tenido lugar en Chile representada por un considerable aumento de enfermedades crónicas no transmisibles relacionadas con el estilo de vida. En la ciudad de Villarrica, se llevó a cabo una intervención educativa de abril a septiembre del año 2002, sobre las conductas que conforman el estilo de vida promotor de salud, de acuerdo a un modelo creado por una enfermera norteamericana de nombre Nola Pender. Esta investigadora creó un instrumento que permite medir "Estilo de Vida Promotor de Salud" (EVPS), el cual tiene seis sub áreas, es decir, autorrealización, responsabilidad de salud, ejercicio, nutrición, apoyo interpersonal y manejo de estrés. El estudio determinó el impacto de la intervención en dos grupos de mujeres. (Carreño, Vyhmeister, Grau, \& Ivanovic, 2006).

Este fue un estudio piloto, de diseño experimental y de cohorte única. La población estuvo compuesta por 300 mujeres, 150 Adventistas del Séptimo Día (MASD, n=150) y 150 No Adventistas (MNASD, $\mathrm{n}=150$ ) del sector urbano de la comuna de Villarrica, novena región de Chile, con edades entre 20-45 años. De la población antes 
mencionada, se seleccionó una muestra aleatoria y representativa de 18 MASD y 18 MNASD. Los resultados indicaron que las medianas aumentaron significativamente entre los períodos de pre- y post-test, tanto en los indicadores totales expresados como EVPS en ambos grupos, (MNASD) y (MASD), así como en las seis dimensiones por separado (Carreño et al., 2006).

Los hallazgos de varios autores sugieren la necesidad de desarrollar, más programas educativos enfocados a prevenir enfermedades crónicas no transmisibles relacionadas con el estilo de vida.

Han pasado seis años desde la intervención, por lo que el objetivo de este proyecto es analizar y determinar si la estrategia comunicativa utilizada en dicha intervención fue eficaz, tanto en lo conceptual, procedimental, actitudinal, como en lo emotivo.

\subsection{Objetivos}

\section{Objetivo General}

a)

El objetivo de este estudio es determinar la efectividad de la estrategia comunicativa, con la cual se implantó e implementó en la comunidad, la intervención educativa relativa a evaluar el EVPS de acuerdo a Pender.

\section{Objetivo Específico}

b)

Determinar la efectividad de la estrategia comunicativa, para cada una de las sub- escalas o factores de vida saludable del EVPS de Pender, tales como autorrealización, responsabilidad de salud, ejercicio, nutrición, apoyo interpersonal y manejo de estrés.

\section{Hipótesis}

c)

El EVPS que presenta la muestra en el período de re-test en el año 2008, luego de seis años de finalizada la intervención educativa, no presenta diferencias significativas, en comparación con el período de post-test del año 2002, producto de la adecuada estrategia comunicativa implementada en dicha intervención.

\subsection{Antecedentes}

Durante el año 2002 se ejecuto el programa de estilo de vida promotor de salud, el cual tuvo como objetivo principal, la modificación de conductas relacionadas con el estilo de vida. Al respecto, se implementó un programa con estrategias de comunicación y educación para la salud, aplicadas a dos grupos de mujeres, con filosofías de vida diferentes, mujeres Adventistas del Séptimo Día y mujeres no Adventistas del Séptimo Día. A manera de diagnóstico, se aplicó el instrumento Estilo de Vida Promotor de Salud de Pender. A continuación se desarrolló el programa 
educativo, que contemplaba charlas expositivas, talleres, usando elementos de multimedia, audiovisuales, consistió en 15 sesiones con charlas expositivas incluyendo en ellas un test diagnostico, en un periodo de tres meses. A esto se agregan 20 sesiones de prácticas aeróbicas, dos veces por semana y, 13 sesiones de prácticas de cocina saludable, una vez por semana. Al término del programa se realizó un pos-test, por lo cual se aplicó nuevamente el mismo instrumento aplicado en el período de pre-test, para determinar si se adquirieron las conductas promotoras de vida saludable. (Carreño et al., 2006).

Los resultados previos a la presente investigación mostraron que las Medianas en el EVPS aumentaron significativamente entre los períodos de pre- y post test, tanto en los resultados totales expresados como EVPS, como en las seis dimensiones por separado, en ambos grupos. En autorrealización, responsabilidad en salud, nutrición, apoyo interpersonal y manejo de estrés. En las MNASD, todos los cambios fueron significativos al nivel de $\mathrm{P}<0.0001$. A pesar del hecho que las MASD presentaron cambios significativos en todos los factores medidos en el EVPS, el progreso de la dimensión nutrición fue menos significativo $(P<0.0005)$ y el puntaje de la dimensión ejercicio fue más bajo $(P<0.0042)$, que aquellos registrados por las MNASD. Ambos grupos, MNASD y MASD experimentaron cambios positivos y significativos en todas las dimensiones del EVPS. Al examinar los valores de las Medianas de las MNASD y MASD en las dimensiones del EVPS durante los periodos de pre- y post-test, con la excepción de la dimensión ejercicio, las MASD tuvieron más alto puntaje en todas las dimensiones del EVPS en el pre- y post-test. (Carreño et al., 2006).

En la dimensión ejercicio, en ambos periodos, las MNASD tuvieron puntajes más altos que las MASD. En el pre-test, el puntaje más bajo de la Mediana fue observado en la dimensión responsabilidad en salud en las MNASD; sin embargo, en el período de post-test, para la dimensión ejercicio en MASD, la Mediana aumentó 0.9 puntos comparado con los 0,8 puntos de las MNASD. En el post-test, las MNASD presentaron cambios menores en comparación con la MASD, pero más altos, en responsabilidad en salud, nutrición y en manejo del estrés. En las MASD, las medianas mostraron mayor cambio de comportamiento, tanto en las dimensiones de estilo de vida promotor de salud, como ejercicio y en apoyo interpersonal En la dimensión autorrealización, los cambios en el post-test fueron similares para ambos grupos (Carreño et al., 2006).

Los valores del test de Wilcoxon, para la comparación de los puntajes en las MNASD vs. MASD, en las dimensiones del EVPS durante los periodos de pre- y post-test. Con la excepción de la dimensión nutrición, no se observaron diferencias significativas en los otros factores del EVPS, tanto en los periodos de pre y post-test. Las MASD registraron puntajes significativamente más altos, para la dimensión nutrición, en comparación con sus pares MNASD, tanto en el periodo de pre- $(p<0.0001)$ como en el de post-test $(p<0.0005)$ (Carreño et al., 2006).

Con relación a los antecedentes previamente expuestos, se postula la presente investigación, con el objeto de evaluar el impacto de la intervención educativa seis años después de su finalización. 


\subsection{Desarrollo del marco epistemológico y conceptual}

Las teorías o los modelos del cambio de comportamiento postulan que la adopción de comportamientos sanos es un proceso en el cual los individuos avanzan, a través de diversas etapas, hasta que el nuevo comportamiento se convierta en parte de la vida diaria. Los modelos recalcan que los mensajes y programas transmitidos por los medios de comunicación son más eficaces en las etapas iníciales, aunque las comunicaciones interpersonales y las redes comunitarias de apoyo social son sumamente importantes durante las etapas posteriores. La información y la comunicación en salud son fundamentales para la adopción de modos de vida sanos, en forma individual y colectiva. "Dado que el comportamiento humano es un factor primordial en los resultados de salud, las inversiones sanitarias deben centrarse tanto en los comportamientos como en los establecimientos de salud y la prestación de servicios. La solución de los problemas de salud requiere que las personas comprendan y estén motivadas para adoptar o cambiar ciertos comportamientos. Por lo tanto, la comunicación eficaz debe formar parte de cualquier estrategia de inversión sanitaria" (Banco Mundial, 1998).

La (OPS) ha tenido una participación destacada en la promoción de la salud y el bienestar, gracias a sus programas de comunicación para la salud. En 1986, la Carta de Otawa para la Promoción de la Salud la definió como "el proceso de facultar a las personas para que aumenten el control que tienen sobre su salud para mejorarla" (Coe, 1998).

Los medios de comunicación son actores claves para la promoción de la salud. La comunicación para la salud es definida como "la modificación del comportamiento humano y los factores ambientales relacionados con ese comportamiento, que directa 0 indirectamente promueven la salud, previenen enfermedades o protegen a los individuos del daño", "un proceso de presentar y evaluar información educativa persuasiva, interesante y atractiva que dé por resultado comportamientos individuales y sociales sanos" ( Coe, 1998 ).

Los elementos claves de un programa de comunicación para la salud son el uso de la teoría de la persuasión, la investigación y segmentación de la audiencia y un proceso sistemático de desarrollo de programas" ( Coe, 1998 ).

Diversos estudios han demostrado que la comunicación interpersonal, juega un papel importante en la difusión de conocimientos, en la modificación o reforzamiento de conductas, valores y normas sociales, que contribuyen al mejoramiento de la calidad de vida. Diversos autores presentan una visión del hecho comunicativo, cuya premisa básica es suponer que ésta, la comunicación, no es un hecho completamente voluntario. Se da la comunicación cuando dos o más personas están en "situación" de relación (Watzlawick, Weakiand, Beavin, Bateson). Esta comunicación es involuntaria, no existe un emisor y un receptor, ambos actúan como tales simultáneamente. Estamos obligados a comunicarnos, dicen los estudiosos de Palo Alto, no podemos huir de la comunicación. 
El concepto de comunicación también lo entendemos de forma participativa, interactiva y de multimediación, lo cual implica que tanto el emisor como el receptor son productores de sentido, no se intercambian mensajes aislados, sino insertos en conjuntos textuales. Esta comunicación necesaria debe apuntar a respetar la cultura de los otros y favorecer los procesos de empoderamiento y resiliencia. Existen diferentes modelos y teorías de la comunicación aplicados en contextos de salud, los cuales han sido ampliamente desarrollados. (Del Valle, 2002)

\section{METODOLOGÍA}

\section{1.- Estudio de la Comunidad}

El estudio inicial se llevó a cabo durante el año 2002, en la comuna de Villarrica, la cual se encuentra ubicada a $87 \mathrm{Km}$. de la ciudad de Temuco, Chile. Cuenta con una población de 45.531 habitantes (INE. 2002) de los cuales $67,8 \%$ (30.859) corresponde a población urbana y el $32,2 \%$ (14.672) a población rural. En las familias de la comuna prevalecen enfermedades como la hipertensión arterial, la diabetes, patologías digestivas y el cáncer, principalmente gástrico. Estas enfermedades crónicas se relacionan con el estilo de vida que se practique y corresponden al principal problema de salud del adulto entre 20 y 65 años en Chile, provocando dos tercios de la mortalidad en este grupo.

\subsection{Diseño del Estudio}

El estudio piloto efectuado el año 2002 fue experimental, comparativo y de cohorte única. El estudio del año 2008, corresponde a un estudio longitudinal y comparativo, entre los resultados del programa EVPS obtenidos en el post-test en el estudio del año 2002 y en el re-test en el año 2008, entre MASD y MNASD.

\subsection{Población y Selección de la Muestra}

La población del año 2002 estuvo compuesta por 300 mujeres, 150 Adventistas del Séptimo Día (MASD, $n=150$ ) y 150 No Adventistas (MNASD, $n=150$ ) del sector urbano de la comuna de Villarrica, Novena región de Chile, con edades entre 20-45 años. Se seleccionó una muestra aleatoria de 18 MASD y 18 MNASD, quienes se enrolaron en el programa EVPS. Ambos grupos fueron comparables, ya que todas las mujeres eran dueñas de casa, tenían a su cargo la responsabilidad del hogar, cuidado de los niños, preparación de los alimentos, pertenecían a un estrato socioeconómico similar (SES) (medio-bajo SES) y un nivel escolar (enseñanza media incompleta). Esta muestra fue objeto de seguimiento en el presente estudio según se indica en la Figura 1 , (en Anexos) la cual muestra que no fue posible ubicar solo a 3 MNASD. 


\subsection{Estudios Efectuados el Año 2002}

\subsubsection{Estrato socio-económico (SES)}

Fue evaluado aplicando el método de Graffar modificado adaptado a la población urbana de Chile, que considera el nivel de escolaridad y ocupación del jefe del hogar, y características de la vivienda (calidad, tenencia, abastecimiento de agua, eliminación de excretas y bienes del hogar). (Álvarez et al. 1985). Esta escala clasifica una población en 6 estratos socioeconómicos: $1=$ Alto; $2=$ Medio alto; $3=$ Medio; $4=$ Medio-bajo; $5=$ Bajo y $6=$ Extrema pobreza.

\subsubsection{Estilo de Vida promotor de Salud ( EVPS )}

El EVPS se determinó de acuerdo al instrumento creado por Nola Pender, junto a otras investigadoras de la Universidad de Massachussets, EE.UU (Walker, Kerr, Pender, \& Sechrist, 1990). Basado en las respuestas de 952 adultos en comunidades del medio oeste de este país, el perfil de Estilo de Vida Promotor de Salud fue evaluado usando análisis de ítem, análisis de factores, y medidas de confiabilidad. El análisis de factores aisló seis dimensiones: Autorrealización, Responsabilidad en salud, Ejercicio, Nutrición, apoyo Interpersonal) y manejo de Estrés. Estos seis factores fueron el $47.1 \%$ de la varianza en la medición de los 48-ítem. Análisis de factor de segundo orden dieron un solo factor, interpretado como Estilo de Vida Promotor de Salud. El coeficiente de confiabilidad Alfa para la escala total es .922; coeficientes alfa para las sub-escalas varían desde .702 a .904. Una evaluación posterior con diferentes poblaciones parece justificarse. Este instrumento capacitará a los investigadores para estudiar modelos y determinantes de estilos de vida promotores de salud, así como los efectos de intervenciones para alterar el estilo de vida.

\subsubsection{Versión en Español del instrumento EVPS}

La versión en español fue luego administrada a un grupo diverso, pero predominantemente mejicano-americano, de 485 hispanos residentes en áreas metropolitanas y rurales que las circundan. En un análisis de factores de componentes principales, todos menos un ítem, pesaron significativamente en seis factores similares a aquellos aislados previamente, durante la evaluación psicométrica de la versión inglesa. Aquellas seis dimensiones comprenden las subescalas del estilo de vida promotor de salud de: auto realización, responsabilidad en salud, ejercicio, nutrición, apoyo interpersonal y manejo del estrés. Los seis factores explicaron el $45.9 \%$ de la varianza en la medida. El análisis de factores de segundo orden entrega un solo factor, interpretado como estilo de vida promotor de salud. El coeficiente de confiabilidad alfa para la escala total fue .93 y 2 semanas después del test-re test, la confiabilidad fue .86; Los coeficientes alfa para las sub escalas estuvieron en el rango .70 a .87.

El instrumento fue elaborado por Walker, Sechrist y Pender en el año 1987, teniendo como base una escala psicométrica formulada por las mismas autoras y 
quienes validaron su versión castellana en el año 1990 (Walker, Kerr, Pender, \& Sechrist, 1990; Walker, Sechrist, \& Pender, 1987).

Esta escala está formada por 48 ítems, en los cuales las mujeres entrevistadas indicaron la frecuencia con la que realizan cada actividad comprendida en ellos. Este instrumento permite valorar el Estilo de Vida Promotor de Salud Total y sus seis dimensiones: Autorrealización, Responsabilidad en Salud, Ejercicio, Nutrición, Apoyo Interpersonal y Manejo del estrés.

La puntuación de los ítems es la siguiente:

$\begin{array}{ll}\text { Nunca }(\mathrm{N}) \text { : } & 1 \\ \text { A veces }(\mathrm{V}): & 2 \\ \text { Frecuentemente }(\mathrm{F}): & 3 \\ \text { Siempre }(\mathrm{S}): & 4\end{array}$

Este instrumento consta de una escala total y seis sub escalas, que incluyen los siguientes ítems:

Estilo de Vida Promotor de Salud: $\quad 1$ al 48

Autorrealización: $\quad 3,8,9,12,16,17,2123,29,34,37,44,48$.

Responsabilidad de salud $\quad 2,7,15,20,28,32,33,42,43,46$.

Ejercicio:

$4,13,22,30,38$

Nutrición:

$1,5,14,19,26,35$.

Apoyo Interpersonal

Manejo del estrés:

$10,18,24,25,31,39,47$.

$6,11,27,36,40,41,45$.

La puntuación para la escala total es calculada con el promedio para las respuestas de la entrevistada a los 48 ítems y, en forma similar, se procede para obtener las puntuaciones de las seis sub escalas.

\subsection{Diseño metodológico del Programa de Estilo de Vida Promotor de Salud (PEVPS)}

El PEVPS fue desarrollado según el diseño metodológico que se indica en la Tabla 1. (Ver Anexos)

\subsubsection{Periodo de Diagnostico o Pre-test}

El objetivo del período de pre-test efectuado el año 2002 fue determinar a manera de diagnóstico, las conductas de EVPS global, como en las seis dimensiones, es decir, Autorrealización, Responsabilidad en Salud, Ejercicio, Nutrición, Apoyo Interpersonal, 
Manejo del Estrés. Para tal efecto, se aplicó el instrumento para medir el EVPS de Pender tanto en las MASD como en las MNASD. (Persky, Chatterton, Van Horn, Grant, Langenberg, Marvin, 1992)

\subsubsection{Intervención Educativa mediante el PEVPS}

El objetivo del PEVPS fue lograr cambios positivos de comportamiento tanto en el EVPS global, como en las seis dimensiones del PEVPS, es decir, en Autorrealización, Responsabilidad en Salud, Ejercicio, Nutrición, Apoyo interpersonal, Manejo del Estrés. Para tal efecto, el PEVPS se desarrolló en ambos grupos, MASD y MNASD, durante tres meses con treinta y tres sesiones de trabajo en cada grupo. EI PEVPS consistió en un programa de Comunicación para la Salud de carácter cognitivoperceptual, en el cual se enfatizó especialmente, los beneficios del aire puro, descanso, luz solar, agua, manejo y control del estrés, responsabilidad en salud y nutrición haciendo uso de materiales audiovisuales para ilustrar las charlas expositivas, así como comunicación interpersonal como refuerzo a la intervención. Se desarrollaron talleres de cocina dos horas por semana, por un período de trece semanas, en las cuales las mujeres practicaron recetas de cocina saludable. Después de las clases teóricas acerca de los beneficios del ejercicio, las mujeres asistieron a gimnasia aeróbica dos veces por semana, cada sesión con una duración de una hora y, con un total de 20 horas de dedicación a esta dimensión.

\subsubsection{Periodo de Evaluación o Post-test}

El período de post-test comenzó tres meses después de finalizado el PEVPS efectuado en el año 2002. El objetivo fue determinar el impacto del PEVPS, en las conductas de EVPS global, como en las seis dimensiones, es decir, Autorrealización, Responsabilidad en Salud, Ejercicio, Nutrición, Apoyo Interpersonal, Manejo del Estrés. Para tal efecto, se aplicó el instrumento para medir el EVPS de Pender, tanto en las MASD como en las MNASD, al igual que en el período de pre-test. (Persky, et al., 1992)

\subsection{Estudios efectuados el año 2008}

\subsubsection{Periodo de Re-evaluación o Re-test}

El período de re-test se efectuó en el mes de diciembre del año 2008, es decir, seis años después de finalizado el PEVPS. El objetivo fue determinar el impacto del programa, en el largo plazo, tanto en las conductas de EVPS global, como en las seis dimensiones, es decir, Autorrealización, Responsabilidad en Salud, Ejercicio, Nutrición, Apoyo Interpersonal, Manejo del Estrés. Para tal efecto, se aplicó el instrumento para medir el EVPS de Pender tanto en las MASD como en las MNASD, al igual que en el período de pre-test y post-test. (Persky, et al., 1992) 


\subsubsection{Análisis Estadístico}

Los resultados serán expresados como mediana, modo, rango y quartiles (PROC UNIVARIATE), utilizando el software Statistical Análisis System Package (SAS, 1983). Este análisis estadístico incluirá la prueba del Signo (PROC UNIVARIATE), para determinar diferencias significativas en cada dimensión del EVPS, separadamente para cada grupo de MASD y MNASD, al aplicar el instrumento después de seis años, a lo menos, al $75 \%$ de la muestra encuestada el año 2002. La prueba de Wilcoxon (PROC NPAR1WAY) se utilizará para comparar los grupos MASD vs. MNASD, durante los periodos de pre-test vs. post-test, post-test vs. re-test. (Kamieneski, Brown, Mitchell, Perrin, Dindial, 2000)

\section{ANÁLISIS Y DISCUSIÓN}

La Tabla 2 (Ver Anexos) muestra los puntajes en el EVPS, como en sus dimensiones, en los períodos de pre-test y post-test obtenidos el año 2002 y, en el período de retest, durante el año 2008 tanto en las MASD como en las MNASD. En ambos grupos, las medianas de los puntajes aumentaron significativamente del periodo de pre-test al de post-test, tanto en los resultados totales expresados como EVPS, como en cada una de las seis dimensiones.

En las MNASD, todos los cambios fueron significativos al nivel de $P<0.0001$. A pesar del hecho que las MASD presentaron cambios significativos en todas las dimensiones medidas en el EVPS, el progreso en las dimensiones nutrición $(P<0.0005)$ y ejercicio $(P<0.0042)$ fue menos significativo que el registrado por las MNASD. En las MASD, al comparar el período de re-test efectuado el año 2008, con el período de post-test efectuado el año 2002 solamente se observó un leve pero significativo descenso en las medianas del EVPS de 3.4 a 3.1 ( $P<0.0309$ ). En el resto de las dimensiones del EVPS no se presentaron diferencias significativas entre el período de re-test con el de posttest, Por el contrario, en las MNASD se observó un descenso significativo en las medianas del EVPS de 3,1 a $2,7(P<0.001)$, en autorrealización, de 3,7 a 3,4 ( $P<$ $0.0018)$, en ejercicio, de 3,0 a $1,6(P<0.0074)$ y en la dimensión manejo de estrés de 3.0 a 2.3 ( $p<0.0129)$; en el resto de las dimensiones, aunque las medianas disminuyeron en el período de re- test, en comparación con el período de post-test, no se observaron cambios significativos.

La Tabla 3 (Ver Anexos) resume los valores del test de Wilcoxon para la comparación de los puntajes en las MASD vs. MNASD, tanto en el EVPS como en sus dimensiones, durante los periodos de pre-test, post-test y re-test. Con la excepción de la dimensión nutrición, no se observaron diferencias significativas en las otras dimensiones del EVPS, tanto en los periodos de pre y post-test. Al respecto, las MASD manifestaron puntajes significativamente más altos para la dimensión nutrición que sus pares MNASD, tanto en el periodo de pre-test $(P<0.0001)$ como en el post-test $(\mathrm{P}<0.0005)$. No obstante, durante el período de re-test, las MASD presentaron medianas significativamente más elevadas, en comparación con las MNASD, tanto en el EVPS ( $P<0.0048)$, como en las dimensiones ejercicio $(P<0.0008)$, nutrición $(P<$ $0.0183)$ y manejo de estrés $(P<0.0381)$. La Figura 2 ilustra los valores de las 
medianas de las MASD y MNASD, tanto en el EVPS, como en sus dimensiones, durante los períodos de pre-test, post-test y re-test.

La Figura 3 muestra que las MASD, tienen una frecuencia de actividades promotoras de vida saludable, es decir un EVPS superior a la de las MNASD ya que desde el pretest obtuvieron puntajes más altos, manifestando cambios y alcanzando medianas más altas en el pos-test como en el re-test, en contraste con las MNASD, quienes presentaron un descenso significativo.

En autorrealización, ambos grupos presentaron puntajes similares en los períodos de pre-test y post-test en el año 2002 y se mantuvieron en su cambio de comportamiento después de seis años, lo que muestra una frecuencia de actividades semejante, alcanzando las MASD un puntaje más alto en el re-test, en esta dimensión. En responsabilidad en salud, las MASD mostraron medianas más altas en el pre-test, post-test y en re-test, y mostraron el mayor descenso luego de seis años después de la intervención en comparación con las MNASD, las cuales muestran una tendencia al descenso. En ejercicio físico, las MNASD el año 2002 comenzaron con puntajes más altos y finalizaron con mayores logros, en los periodos de pre-test y post-test, siendo la única dimensión en donde las MASD obtuvieron puntajes más bajos; sin embargo después de seis años, el re- test muestra que las MNASD bajaron significativamente sus puntajes en contraste con las MASD, las cuales han mantenido sus logros, ya que no tuvieron un cambio significativo en esta dimensión. En nutrición, las medianas muestran que las MASD tienen un puntaje alto en los periodos de pretest, post-test y re-test.

En el caso de las MNASD comenzaron con medianas más bajas y, por la intervención educativa, obtuvieron en el período de post-test, medianas similares al registrado por las MASD en el período de pre-test. Las medianas del re-test efectuado en el presente estudio muestra que las MNASD no han logrado mantener los cambios en esta dimensión a diferencia de las MASD, quienes mantienen conductas favorables en esta dimensión. En apoyo interpersonal, las MASD y as MNASD presentaron en el pre-test valores iguales en sus medianas; sin embargo, tanto en el período de post-test, como en el período de re-test seis años después, las medianas muestran que en las MASD los cambios de conducta permanecen en el tiempo después de la intervención. En cuanto al manejo de estrés, las medianas muestran que las MASD, tienen puntajes más altos en los periodos de pre-test, post-test, y el re-test seis años después, en contraste con las MNASD, cuyo puntaje sufrió un descenso considerable.

Es indudable que el enfoque de promoción de la salud es reconocido hoy mundialmente, como la estrategia de alto rendimiento costo-beneficio para mejorar la salud de los individuos. Cuando el cliente es la familia o la comunidad, tiene un valor agregado. En estas situaciones, tanto la familia como la comunidad son responsables del desarrollo de la salud de las futuras generaciones, porque es en este contexto que se transmiten valores, actitudes y conductas relacionadas con la salud, resumidas en estilos de vida familiar saludables capaces de reducir situaciones de riesgo. 
Diversos estudios llevados a cabo en Chile y también en el extranjero, en diferentes grupos etarios, han enfatizado los beneficios que representa la implementación de programas de promoción de la salud en el logro de un estilo de vida saludable. (OPS, 1996; Pender, et al., 2001; Jauregui, et al., 1998; Willett, 2003; Foster, 1989; WHO,2004; OMS, 1990; Burdine, et al., 1984; Reynolds, et al., 1998 ; Reynolds, et al., 2000; Donnelly, et al., 1996; Singh, Fraser, 1998; Fraser, Shavlik, 1997; Fraser, Sumbureru, Pribis, Neil, Frankson, 1997; Singh, Fraser, Knutsen, Lindsted, Bennett, 2001; Chile, INE, 2002; The World Medical Association, 1964; Alvarez, Muzzo, Ivanovic, 1985; Walker, 1990)

Al observar los resultados del estudio efectuado en el año 2002, así como los indicadores del estudio del año 2008, nos damos cuenta que las MASD, tienen una frecuencia de actividades promotoras de salud, es decir un EVPS superior a la de las MNASD y, en general, en todas las dimensiones. Este hecho podría explicarse debido a la filosofía de vida de las MASD y a la educación que reciben por parte de su Iglesia, ya que con frecuencia asisten a charlas y conferencias en las cuales se usan elementos didácticos de multimedia que dan énfasis a la salud, independiente de su nivel socio económico, ya que en sus creencias existe el concepto de salud integral, en las áreas: física, mental, social y espiritual. Diversos estudios efectuados a nivel internacional han destacado el positivo impacto que tiene el estilo de vida saludable de carácter integral que practica la comunidad de los Adventistas del Séptimo Día, en la prevención de las enfermedades crónicas no transmisibles relacionadas con la dieta. (Fraser, et al., 1997; Fraser, Sumbureru, Pribis, Neil, Frankson, 1997; Singh, et al., 2001; Chile, INE, 2002; The World Medical Association, 1964; Alvarez, et al.,1985)

En autorrealización, ambos grupos presentaron puntajes similares en los períodos de pre-test y post-test en el año 2002 y se mantuvieron en su cambio de comportamiento después de seis años lo que muestra una frecuencia de actividades semejante, alcanzando las MASD puntaje más alto en esta dimensión. Este hecho se explicaría en las MASD, por las diversas actividades que realizan a favor del prójimo, como asistencia social y, en el caso de las MNASD, contribuyó el hecho que realizaban mayor ejercicio físico el año 2002 que las MASD, lo que tuvo mucha ingerencia en su autorrealización.

En responsabilidad en salud, las MASD mostraron medianas más altas en el pre-test, post-test y en re-test en comparación con las MNASD, probablemente debido a la conciencia de cuidado de su cuerpo que adquieren, producto de su filosofía de vida y educación integral que reciben de su Iglesia, lo que ha quedado de manifiesto en diferentes estudios a nivel internacional. (Singh, et al., 1998; Fraser, et al., 1997; Fraser, Sumbureru, et al., 1997; Singh, et al., 2001; Chile, INE, 2002; The World Medical Association, 1964; Alvarez, et al., 1985; Walker, 1990)

Sin embargo mostraron el mayor descenso luego de seis años después de la intervención, es probable que esto se deba a la disminución de frecuencia de visitas al médico y otros profesionales de los centros de atención primaria, debido a las demoras en la atención. Las MNASD manejaban poco conocimiento sobre salud preventiva y comportamiento saludable. Sin embargo, aunque se constató un 
descenso en las medianas entre los períodos de post-test y re-test, tendieron a mantener a lo largo de seis años los cambios positivos en esta dimensión. Es probable que esto se deba a las orientaciones recibidas en el PEVPS, ya que al no tener acceso a información y visitas periódicas de profesionales de la salud, como lo es en el caso de las MASD en sus iglesias y reuniones, las MNASD valoran la entrevista con profesionales del área médica, cuando presentan síntomas de posible enfermedad o anomalías, acudiendo a los consultorios y en algunos casos haciendo el esfuerzo económico de visitar en forma particular al médico.

En ejercicio físico, las MNASD el año 2002 comenzaron con puntajes más altos y finalizaron con mayores logros, siendo la única dimensión en donde las MASD obtuvieron puntajes más bajos; sin embargo después de seis años, las MNASD bajaron significativamente, en contraste con las MASD, las cuales no tuvieron un cambio significativo en esta dimensión. Las MNASD comenzaron con puntajes altos como lo muestra el pre-test el año 2002, debido a que en ese tiempo, ellas estaban participando en bailes folklóricos, de 2 a 3 veces por semana en forma sistemática, preparando una presentación para el mes de septiembre, lo que hizo aumentar sus puntajes.

En el caso de las MASD, en el PEVPS adquirieron el habito de hacer ejercicio y 0 caminatas aeróbicas en forma permanente, lo que aumento el puntaje en el post test y lo han mantenido en el re-test aplicado en el año 2008. Es así como en ejercicio físico, las medianas muestran que las MASD están por debajo de las MNASD, lo que podría explicarse por la vida sedentaria de las MASD previo a la intervención; si bien es cierto existía el conocimiento, no se le había dado la debida importancia. Las MNASD comenzaron obteniendo puntajes más altos en la dimensión ejercicio físico, debido a que previo a la intervención, ellas ya estaban participando sistemáticamente, dos a tres veces por semana, de un programa de actividad física; sin embargo, el retest efectuado el año 2008 muestra que los cambios favorables no se han mantenido en el tiempo en las MNASD, no así en las MASD, que muestran medianas más altas seis años después de la intervención.

En nutrición, las medianas muestran que las MASD tienen una dieta muy saludable, ya que muchas de ellas declararon tener un régimen ovo-lacto vegetariano, bajo en calorías y grasas animales y rico en fibra dietaría. Se puede observar que tenían conocimiento previo, en relación a las características de una dieta saludable y que, por la intervención educativa, hubo también un cambio favorable de comportamiento. En el caso de las MNASD comenzaron con medianas más bajas y, por la intervención educativa, obtuvieron en el período de post-test, medianas similares al registrado por las MASD en el período de pre-test.

Este hecho manifiesta el positivo impacto de la intervención educativa producto del desarrollo del PEVPS en ambos grupos, siendo especialmente beneficioso en las MNASD, las cuales al inicio del programa, no tenían los conocimientos suficientes que se tradujeran en conductas apropiadas para seleccionar una dieta saludable. Las medianas del re-test efectuado en el presente estudio muestra que las MNASD no han logrado mantener los cambios en esta dimensión a diferencia de las MASD, las 
cuales si han mantenido los cambios en este comportamiento saludable. Esto se debe a que no solo es necesario manejar información, conocimiento, de conductas promotoras de vida saludable, también se hace necesario mantener un refuerzo en forma periódica, que permita mantener el cambio de conducta, lo que se da en este caso en la MASD, por medio del soporte de la religión. Los beneficios de una alimentación saludable han quedado de manifiesto en varios estudios efectuados en comunidades Adventistas del Séptimo Día. (Fraser, et al., 1997; Singh, et al., 2001; Chile, INE, 2002; The World Medical Association, 1964; Alvarez, et al., 1985; Walker, 1990)

En apoyo interpersonal, ambos grupos presentaron en el pre-test valores iguales en sus medianas; sin embargo, tanto en el período de post-test, como en el período de re-test seis años después, las medianas muestran que en las MASD no sólo hubo mayor cambio sino que también estos cambios de conducta permanecen en el tiempo después de la intervención, aunque en las MNASD también se observa una buena internalización de estas conductas. Esto puede deberse a la red de apoyo que encuentran las MASD, en la comunidad religiosa y a las relaciones afectivas que se desarrollan allí.

En cuanto al manejo de estrés, las medianas muestran que las MASD, en la frecuencia de actividades, manejan mejor el estrés que las MNASD. Después de la intervención educativa, en el post-test, donde se les enseño a controlar el estrés, así como en el re-test seis años después, las MASD presentaron valores superiores en sus medianas, en relación a las MNASD, lo que podría deberse a la red de apoyo, estilo de vida y fe, como ya se ha mencionado.

Los resultados del presente estudio permiten concluir que un programa educativo de promoción de la salud basado en el modelo de Pender, con la estrategia comunicativa adecuada, podría ser de gran utilidad en el mejoramiento de la calidad de vida de la población. ( Bateson, 1972; Bateson, Hall, Watzlawick, 1990; Del Valle, \& Matus, 2007; Eco, 1994; Eco, 2000; López, Parada, Simonetti, 1991; Mucchielli,

1998; Coe, 1998) El programa permite potenciar cada dimensión citada por la autora, lo que lo hace un programa integral, para promover aquellas conductas que fortalecen la salud y abandonar aquellas que la deterioran. Por otra parte, estos programas deberían impartirse desde la edad escolar, para evitar la alta prevalencia de las enfermedades crónicas no transmisibles en la edad adulta, en donde impartir programas se hace más difícil.( Walker, et al., 1987; Walker, et al., 1988)

Estos hallazgos se consideran importantes para el Sector Salud, ya que la implementación de programas de promoción de la salud, como el que se propone en el presente estudio, podrían ser de gran utilidad en la prevención de las enfermedades crónicas no transmisibles relacionadas al estilo de vida.

El EVPS que presenta la muestra en el período de re-test en el año 2008, nos permite apreciar que en ambos grupos MASD y MNASD fueron significativas las diferencias en las medianas, constatándose un descenso, menos significativo en las MASD, en 
comparación con las MNASD, sin embargo el resultado es muy positivo en las dimensiones del EVPS en las MASD, ya que no hay diferencias significativas con el periodo de post test del año 2002.

Desafortunadamente, en las MNASD, hay un descenso en las medianas del EVPS y especialmente en las dimensiones, autorrealización, ejercicio, y manejo de Estrés, las cuales en el re-test descendieron a niveles del pre-test, esto se debe probablemente a la falta de refuerzo en el tiempo, de los beneficios que para la salud representa, el mantener las mencionadas conductas, situación que no ocurre en las MASD, ya que la filosofía de la iglesia, permanentemente enfatiza estos aspectos. El ambiente, que funciona también como red de apoyo, ya que en las frecuentes reuniones en conjunto son animados por medio de charlas y seminarios a adquirir y mantener conductas saludables que forman parte del estilo de vida promotor de salud.

También es importante destacar que pese a esta desventaja, de no tener un refuerzo en forma sistemática, así como el acceso a una red que pudiera servir de apoyo al comportamiento adquirido el año 2002, luego de la intervención, las MNASD lograron mantener sus conductas promotoras de vida saludable en autorrealización (aunque el descenso de las medianas fue significativo, el puntaje logrado es alto), responsabilidad en salud, nutrición y apoyo interpersonal.

\section{CONCLUSIONES}

Al analizar el impacto del PEVPS seis años después, se observa en las MASD un alto nivel de efectividad de la estrategia comunicativa del programa, al comparar el período de re-test efectuado el año 2008, con el período de post-test efectuado el año 2002. Por lo tanto, se advierte, tanto en el EVPS, como en las diversas dimensiones, la mayor efectividad de la estrategia comunicativa del programa, en las MASD, lo que nos lleva a aceptar solo en parte la hipótesis formulada en el presente estudio, que señalaba que "El EVPS que presenta la muestra en el período de re-test en el año 2008, luego de seis años de finalizada la intervención educativa, no presenta diferencias significativas, en comparación con el período de post-test del año 2002, producto de la adecuada estrategia comunicativa implementada en dicha intervención". La presente investigación nos permite apreciar que en ambos grupos MASD y MNASD fueron significativas las diferencias en las medianas, constatándose un descenso, menos significativo en las MASD, en comparación con las MNASD.

Esto nos permite concluir que la estrategia comunicativa empleada en la intervención, si bien es cierto, fue importante para el cambio de comportamiento, el año 2002 permitiendo a las personas adoptar conductas promotoras de vida saludable, tanto en las MASD, como en algunas dimensiones del EVPS en las MNASD, sin embargo son estas últimas, quienes mostraron un descenso significativo en el EVPS, como en algunas dimensiones. Esto pone de manifiesto la importancia de un refuerzo de estas conductas en forma permanente, el cual a través de la práctica de la religión, las MASD mantienen estas conductas en el tiempo. 
Esto no ocurrió en las MNASD, por lo que se sugiere que un PEVPS debiera contemplar seguimientos frecuentes y refuerzos periódicos, que conlleven a la conservación de un EVS en el tiempo.

\section{REFERENCIAS}

Alvarez M., Muzzo S.\& Ivanovic D.(1985). Escala para medición del nivel socioeconómico en el área de la salud. Rev Med Chil;113:243-9.

Arthur L., Pena M., Holbert D.(2001). Effects of socioeconomic status on the obesity knowledge of adolescents from six Latin American cities. Int J Obes Relat Metab Disord , (258): 1262-8.

Bateson, G. (1972). Pasos hacia una ecología de la mente: colección de ensayos en antropología, psiquiatría, evolución y epistemología. New York: Ballantine Books.

Bateson, Hall, W. (1990). La nueva comunicación. España: Cairos

Burdine J. \& et al. (1984). The effects of ethnicity, sex and father's occupation on heart health knowledge and nutrition behaviour of school children: the Texas youth health awareness survey. J Sch Health, (542): 87-90.

Carreño, J., VYhmeister, G., Grau, L., \& Ivanovic D. (2006). A health promotion program in Adventist and non-Adventist women based on Pender's model. Public Health, $120(4):$ 346-355

Coe, G. (1998). Comunicación y Promoción de la Salud. Revista Latinoamericana de Comunicación Chasqui, (63)

Donelly J., Jacobsen D., Whatley J., Hill J., Swift L., Cherrington A., et al.(1996) Nutrition and physical activity program to attenuate obesity and promote physical and metabolic fitness in elementary school children. Obes Res, (4):229-43.

Eco, U. (1994). Signo. Barcelona: Editorial Labor.

Eco, U. (2000). Tratado de Semiótica General. Barcelona: Editorial Lumen.

FAO/OMS. (1992). Conferencia Internacional sobre Nutrición: Nutrición y DesarrolloEvaluación General. Roma.

Fraser G., Shavlik D.(1997). Risk factors for all-cause and coronary heart disease mortality in the oldest-old. The Adventist Health Study. Arch Intern Med (157):224958.

Fraser G., Sumbureru D., Pribis P., Neil R., Frankson M.(1997). Association among health habits, risk factors, and all-cause mortality in a black California population. Epidemiology 1997 (8) :168-74. 
Jauregui, C., Suarez, P.(1998). Promoción de la Salud y Prevención de la Enfermedad, Enfoque en Salud Familiar. Colombia:Editorial Médica Panamericana.

Kamieneski R., Brown C., Mitchell C., Perrin K., Dindial K.(2000). Health benefits achieved through the seventh-day Adventist wellness challenge program. Altern Ther Health Med , (6):65-9

Lopez, A. Parada, A y Simonetti, F.(1991). Introducción a la psicología de la comunicación .Santiago: Ediciones Pontificia Universidad Católica de Chile.

MINISTERIO DE SALUD, Chile.(1998). Lineamientos estratégicos para la promoción de la salud: Más y mejor salud con la gente. Santiago: MINSAL

OMS.(1990). Prevención en la niñez y en la juventud de las enfermedades cardiovasculares del adulto. Serie de Informes Técnicos 792. Ginebra

OMS. (1996). Promoción de la Salud: Una antología. Publicación científica No. 557.Washington, DC: Oficina Sanitaria Panamericana.

Pender, N., Murdaugh C., Parson M.(2001). Health promotion in nursing practice. Englewood Cliffs, NJ : Prentice-Hall.

Persky V., Chatterton R., Van Horn L., Grant M., Langenberg P., Marvin J. (1992) Hormone levels in vegetarian and non vegetarian teenage girls: potential implications for breast cancer risk. Cancer Res (52):578-83.

Reynolds K., Raczynsky J., Binkley D., Franklin F., Duvall R., Devane-Hart K.(1998) Design of 'High 5': a school-based study to promote fruit and vegetable consumption for reduction of cancer risk. J Cancer Educ (13):169-77.

Reynolds K., Franklin F., Binkley D., Raczynski J., Harrington K., Kirk K. (2000) Increasing the fruit andvegetable consumption of fourth-graders: results from the high 5 project. Prev Med, 30:309-19.

Singh P., Fraser G.(1998). Dietary risk factors for colon cancer in a low-risk population. Am J Epidemiol(148):761-74.

Singh P., Fraser G., Knutsen S., Lindsted K., Bennett H.(2001). Validity of a physical activity questionnaire among African_American seventh-day adventists. Med Sci Sports Exerc (33): 468-75.

Singh P., Sabate J., Fraser G.(2003). Does low meat consumption increase life expectancy in humans? Am J Clin Nutr 78(Suppl.): 526S-32S

Singh P., Tonstad S, Abbey D., Fraser G.(1996). Validity of selected physical activity questions in white seventh-day adventists and non-adventists. Med Sci Sports Exerc (28): 1026-37. 
Walker S., Kerr M., Pender N., Sechrist K.(1990) A Spanish language version of the Health-Promoting Lifestyle Profile. Nurs Res (39):268-73.

Walker S., Sechrist K., Pender N. (1987).The Health-Promoting Lifestyle Profile: development and psychometric characteristics. Nurs Res, 36:76-81.

Walker S., Volkan K., Sechrist K., Pender N.(1988) Health promoting life styles of older adults: comparisons with young and middle-aged adults, correlates and patterns. ANS Adv Nurs Sci (11): 76-90.

Willett W. (20003).Lessons from dietary studies in adventists and questions for the future. Am J Clin Nutr ; 78(Suppl.):539S-43S.

Wong C., WONG S., PANG W., Azizah M., Dass M.(2003). Habitual walking and its correlation to better physical function: implications for prevention of physical disability in older persons. J Gerontol A Biol Sci Med Sci (58):555-60

World Health Organization.(2004). 57th world health assembly, 17-22 May 2004. Genova. 


\section{APÉNDICES}

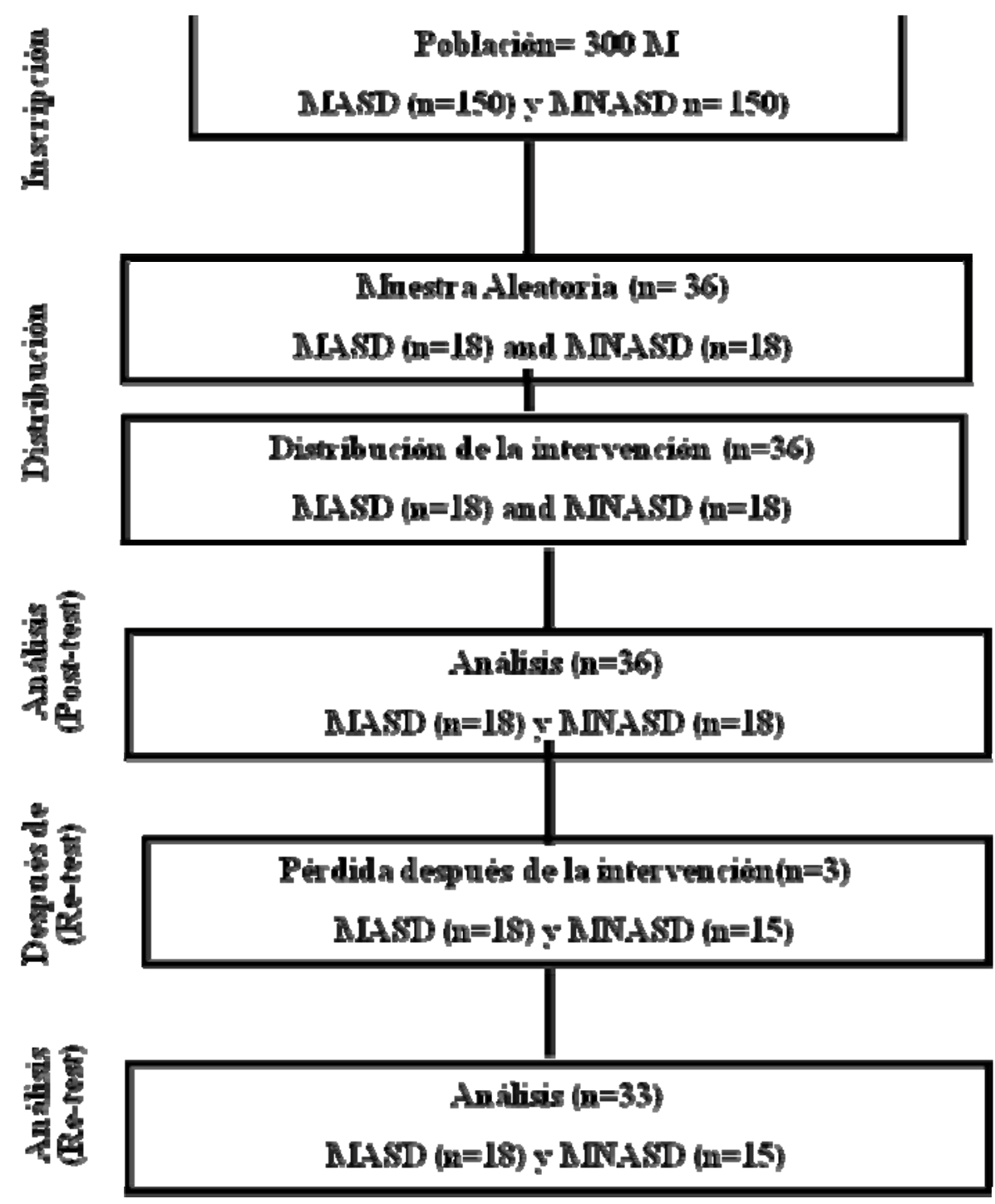

Figura 1. Diagrama de flujo de los sujetos seleccionados en la muestra y tasas de participación durante el Programa de Estilo de Vida Promotor de Salud basado en el Modelo de Pender. $M=$ mujeres; MASD, mujeres Adventistas; MNASD, mujeres no Adventistas del Séptimo Día. 


\section{MASD}

MNASD

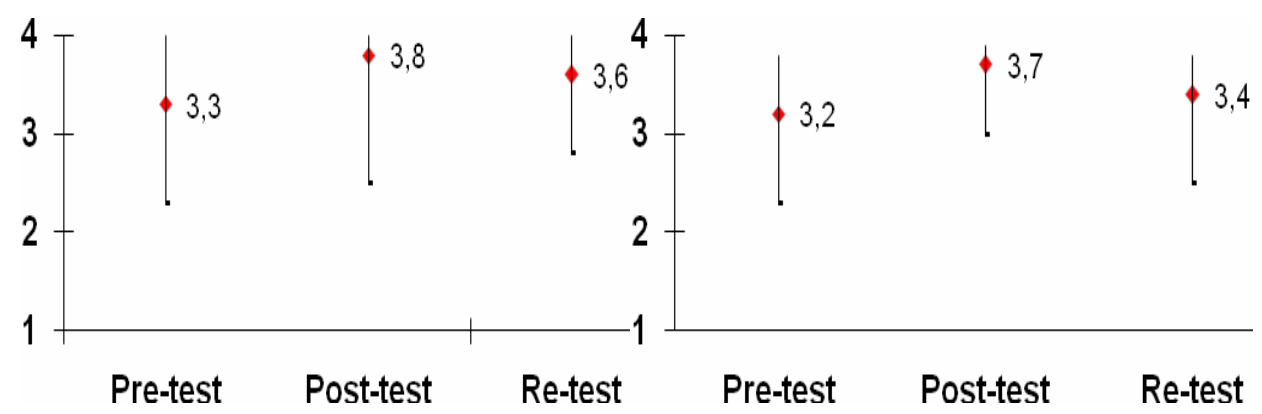

Autorealización

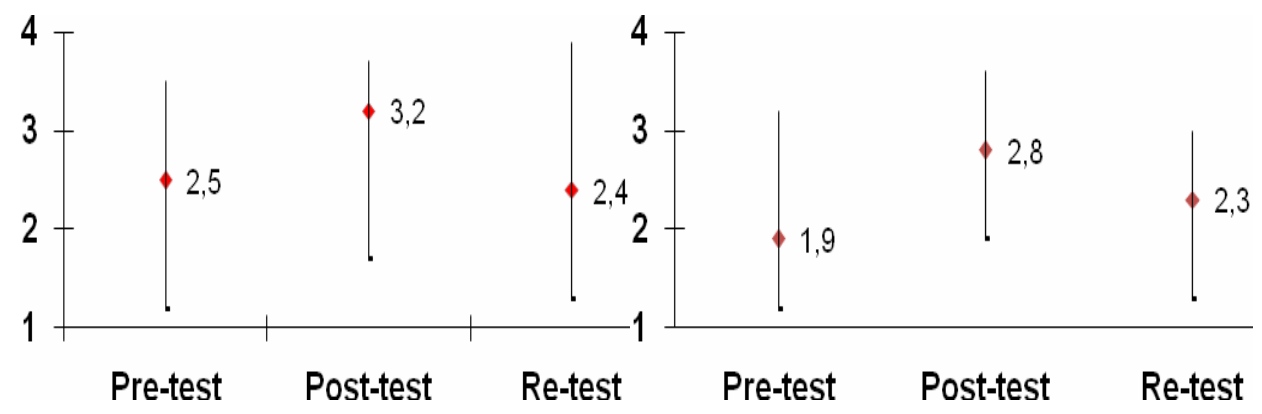

Responsabilidad en Salu

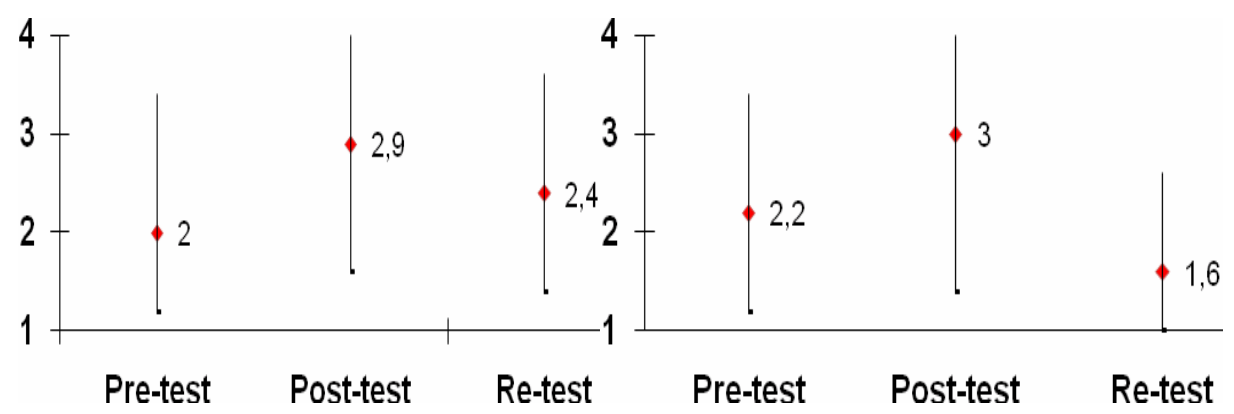

Ejercicio

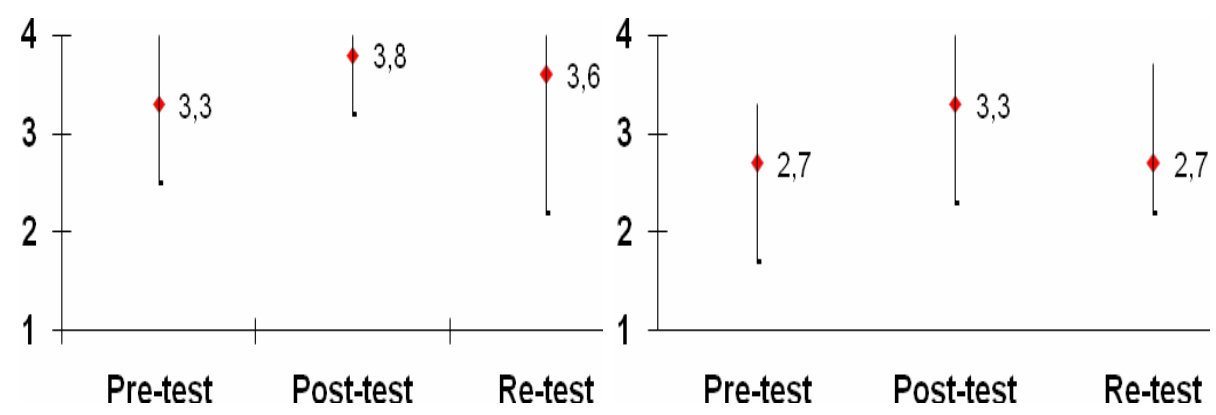

Nutrición 


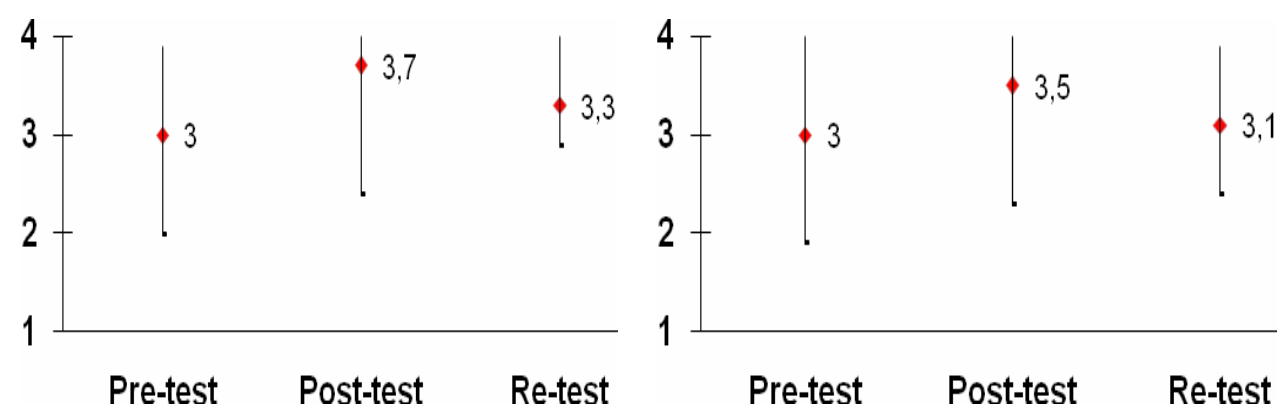

\section{Apoyo Interpersonal}

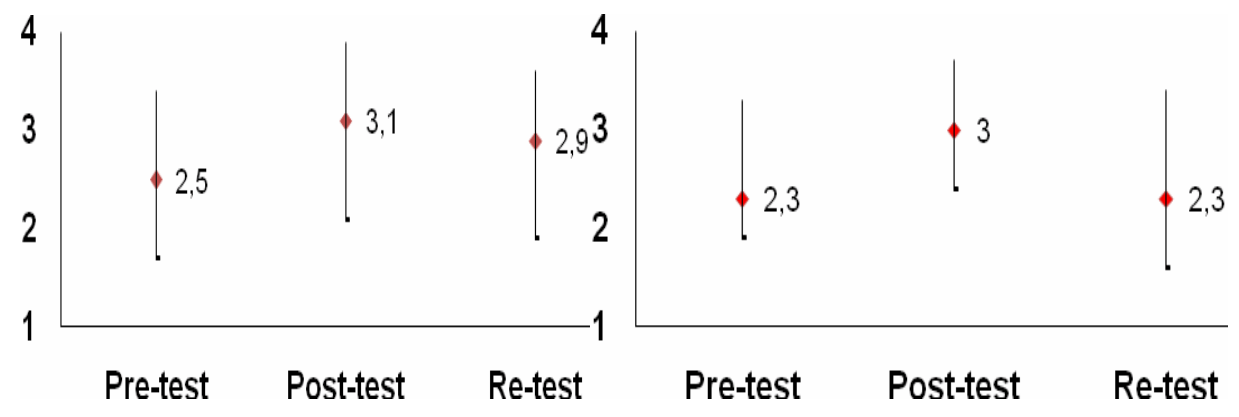

\section{Manejo de Estrés}

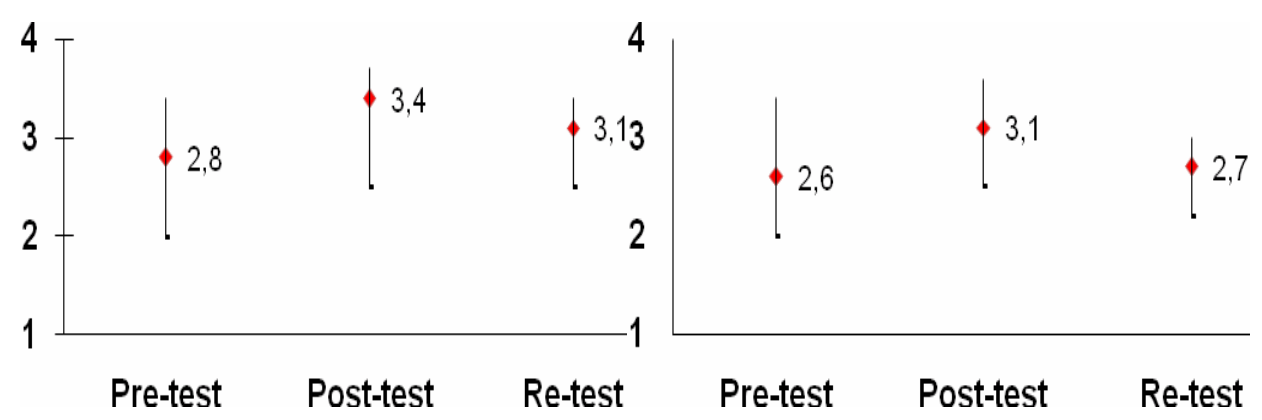

\section{EVPS}

Figura 2. Valores de las medianas y rangos de los resultados totales como de las seis dimensiones del Programa Estilo de Vida Promotor de Salud (PEVPS) en los periodos de pre-test, post-test y re-test en Mujeres Adventistas del Séptimo día (MASD) y Mujeres No Adventistas del Séptimo Día (MNASD). 